\title{
An $E$-service-Based Framework for Inter-administration Cooperation
}

\author{
Mariangela Contenti ${ }^{1}$, Alessandro Termini ${ }^{2}$, Massimo Mecella $^{2}$, and \\ Roberto Baldoni ${ }^{2}$ \\ 1 Università di Roma LUISS "Guido Carli" \\ Centro di Ricerca sui Sistemi Informativi \\ mcontenti@luiss.it \\ 2 Università di Roma "La Sapienza" \\ Dipartimento di Informatica e Sistemistica \\ \{termini,mecella, baldoni\}@dis . uniroma1.it
}

\begin{abstract}
In order to provide electronic service delivery, several activities involving different public agencies need to be related and carried out in coordinated manner, thus resulting in a cooperative process. Currently, $e$-Service technologies seems to offer the enabling infrastructure for supporting cooperative process enactment, even at inter-country level. In this position paper, we outline the architecture we are proposing in the EU-PUBLI.com project, in which orchestration of e-Services constitutes the basis for provision of $e$-Government services. We discuss how such an architecture could support a form, although simple, of management of the cooperative process knowledge.
\end{abstract}

\section{Introduction}

In many countries, laws and administrative provisions usually fragment all the aspects of the life of a citizen according to sectors (i.e. taxation, health, labor, etc.): different responsibilities are then assigned to different agencies of the Public Administration (PA). This fragmented assignment of responsibilities produces difficulties in delivering services to citizens, as such services often result composed by several activities interleaved within complex business processes and involving different agencies. Therefore, in order to be able to provide services satisfactory from the point of view of a citizen agencies should make huge efforts, in term of knowledge and practice of the business processes.

In past years, some experiences (e.g., [1]) have shown that the introduction of consolidated distributed object technologies within the information systems of the agencies represents a mean to render accessible all the data and information stored in their own legacy systems. The proper design and implementation of wrappers allow to abstract from the data physical representation (access wrapper) and to create new information as new relations among the stored data (integration wrapper) [2].

But the adoption of different solutions by different agencies in terms of distributed object platforms and semantic frameworks have hampered interoperability among the different information systems, thus resulting in poor cooperation among their employees and a scarce knowledge management.

The original version of this chapter was revised: The copyright line was incorrect. This has been corrected. The Erratum to this chapter is available at DOI: 10.1007/978-3-540-44836-5_33 
Emerging technologies such as $e$-Services and XML can currently represent the mean to overcome PA boundaries and can offer the technological and conceptual solution to design and to implement cooperation among employees belonging to distinct agencies. In the EU-PUBLI.com project, an approach based on macro-processes [I] and orchestration of $e$-Services [3] is being proposed and experimented, in order to enable cooperation and knowledge management among agencies belonging to different European countries.

A macro-process is a complex business process involving different agencies; $e$-Service technologies can be used to (semi-)automate macro-processes through cooperative applications, thus obtaining cooperative processes, which are enacted in order to offer added-value services to employees, citizens and businesses. Enactment of the cooperative processes is obtained by suitably orchestrating $e$ Services offered by different cooperating agencies.

Such an approach does not necessarily require initial radical modifications either in the macro-process structure nor in the organization internal processes: each agency interfaces the others by offering specific $e$-Services, independently on how it realizes them, its autonomy in changing its own processes is therefore guaranteed. Internal changes do not impact on the macro-process, as they are hidden by the service interfaces exported towards other agencies.

In such an approach, knowledge management, specifically focused on cooperative processes, is enabled and enforced in two specific ways:

- all the information collected in the analysis phase, aimed to identify the macro-process, produces a coherent and homogeneous documentation of the business practices that are often scattered and never formally documented; this is especially true in an inter-country $e$-Government scenario, as the one we are experimenting. Then such a documentation can both (i) be offered to citizens and employees through informative $e$-Services, and, (ii) formalized in an appropriate manner, it is stored as part of the supporting architecture and drives the orchestration of the different $e$-Services. This is especially true in the case of the mappings among different legal frameworks;

- orchestration of $e$-Services (i.e., the enactment of the cooperative processes) produces information on effective run-time executions, exceptions, bottlenecks, etc.; such information can be analyzed and mined in order to infer new process knowledge (business process intelligence, [4]).

In this paper we outline, in Section 2, some of the organizational aspects concerning the emerging need of cooperation and knowledge management; in Section 3 we will present the technologies on which our approach is based. In Section 4 first we briefly describe the EU-PUBLI.com project, then the novel architecture proposed in the project, and in Section [5] we will approach, on the basis of previous and current experiences, some aspect concerning knowledge management and we highlight the architectural subsystems of our architecture specifically supporting it. In Section [6] we compare with related work and finally Section 7 concludes the paper by remarking open issues and future research directions. 


\section{Organizational Issue}

As defined in [5], "a stakeholder in an organization is any group or individual who can affect or is affected by the achievement of the organization's objective". A good organization should then identifies and strengthens its strategies for satisfying as a whole the often conflicting needs and claims of its different stakeholders. The dynamically changing actions a firm performs to comply the stakeholder theory have been then recognized as the mean for guaranteeing the survival and the maintenance of a sustainable competitive advantage for the firm itself.

Although, differently from private-sector firms, the PA's primary mission is to operate on behalf of citizens to pursue public interests and they are by their own nature monopolistic organizations, one of the PA's fundamental task is the provision of public services: the environment turbulence, changing the firms' approach towards a wider customer-oriented flexibility, is influencing the PA's approach, too. Also, the several stakeholders of a public agency are substantially incarnate by citizens, as they play both the role of "investors" (through the tax payment) and of "customer" (as service user) of the PA's actingl.

This implies the the past and consolidated vertical configuration of the PA's, that, focusing on the efficiency rather than on the effectiveness, allowed to reach economy of scale and concentrate all the experiences and knowledge in specific sectors, is currently moving towards different aims and different configurations, driven by the citizens-centric view of service delivery. The new context implies renewing a wide range of operative business processes as often: (i) fragmentation of responsibilities translates in fragmentation of complex processes in atomic administrative activities, each of which assigned to different specific organizational units; (ii) the lack of integration implies frequent interruptions of processes inside each administration resulting in inefficient provision of services, as it often requires that citizens serves as messenger, providing the information needed to establish the communications required.

To design and implement new operative procedures both cost saving and citizen-oriented, is then necessary a new approach which binds together the efficiency and effectiveness of the service delivery: the Information and Communication Technologies (ICT) progress could represents the enabling technological instrument to sustain these efforts, at least for those services characterized by an high information intensity [6].

In this context, the Australian National Audit Office (ANAO) recently published a report focused on the opportunities and challenges that PA should evaluate for delivering services via electronic channels [7]. From the information collected via a questionnaire filled by 66 Commonwealth agencies emerged the main beneficiaries of IT initiatives are individuals and government agencies and the more suitable technology is Internet: the introduction of Internet should reduce the cost and improve the quality of the services. Specifically, the report has identified a framework according to which an agency offering services through

${ }^{1}$ With this view we are neglecting many other stakeholders, e.g., the employees which instead should be taken into account as they could represents, in some cases, a source of resistance to the change. 
the Internet can be in one of four stage, as depicted in Figure 1 each stage represents a different level of service delivery: the higher the stage, the higher the technological and organizational complexity as well as the range of electronically provided services.

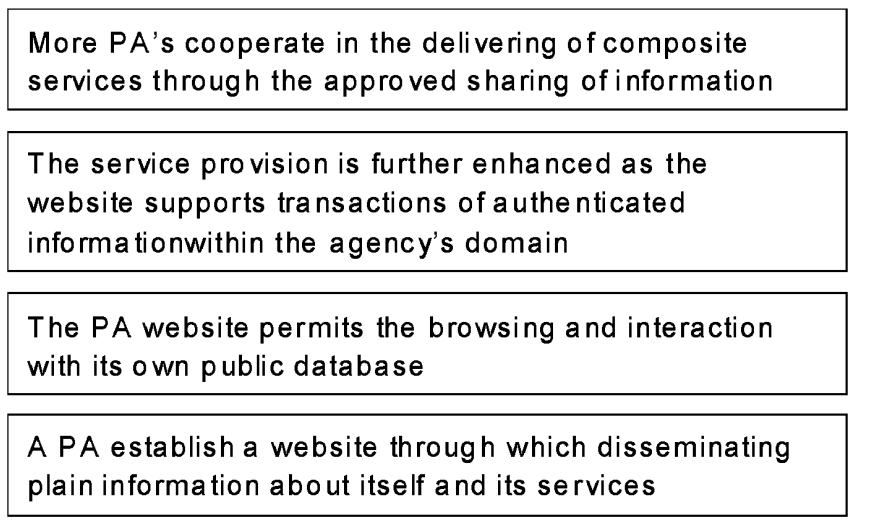

Stage 4 (cooperation)

\section{Stage 3} (service web)

Stage 2 (data web)

Stage 1 (web)

Fig. 1. Our elaboration from the ANAO report [7]

Such a framework has been adopted by the British Central IT Unit of the Cabinet Office in an e-Government benchmark 8], focused on the comparison of the progress status in several sectors among G7 and other leading nations: many countries are positioning their public sectors at stage 2 and 3 , whereas only few countries, i.e., USA, Canada and Singapore, have reached stage 4 and only in few sectors (only in e-Procurement). Such studies confirm the difficulties in establishing a stage $4 e$-Government initiative; such difficulties stem mainly from organizational issues rather than technological ones, as laws and administrative provisions strongly constraint the cooperation processes. Indeed stage 4 requires that agencies identify cooperative processes, that is complex processes involving and "crossing" them in order to furnish added-value services to citizens and businesses [1].

Based on our past experiences, we are convinced that a viable approach to the design of public administration services at stage 4 implies, rather than deep business process reengineering, macro-process technological improvement and this is the one we have adopted in the EU-PUBLI.com project.

In a deep business process reengineering approach, redundant processes in specific organizational units would be eliminated, and some activities would be re-assigned to new organizational units: this would eliminate many information exchanges, thus addressing the main issue of the excessive fragmentation of responsibilities among agencies. Unfortunately, certain issues hamper largescale radical changes in the short and medium term, such as the impossibility of assigning new legal responsibilities to given organizational units (due to the 
difficulty of changing existing laws), the lack of specific skills and resources in some agencies, the time needed to create such skills, and so on.

In the technological improvement approach, each agency is seen as a domain with a proper information asset, made available as data and application services exported on such data; separate agencies are loosely coupled as each agency interfaces the others by offering specific services, independently from their realization; the reengineering of an agency's internal processes does not impact the cooperative processes and the former improvement and enrichment enable the way to more radical macro-process reengineering.

\section{Technological Background}

As the EU-PUBLI.com architecture is based on $e$-Services, in the current section we briefly describe such a paradigm and related technologies. $e$-Services, also referred to as Web-Services, are Web-enabled applications exported by different organizations consisting of well-defined functionalities that allow users and applications to access and perform tasks offered by back-end business applications.

A Service Oriented Architecture (SOA, [9]) is a framework for $e$-Services consisting of (i) some basic operations and (ii) roles, as shown in Figure 2 .

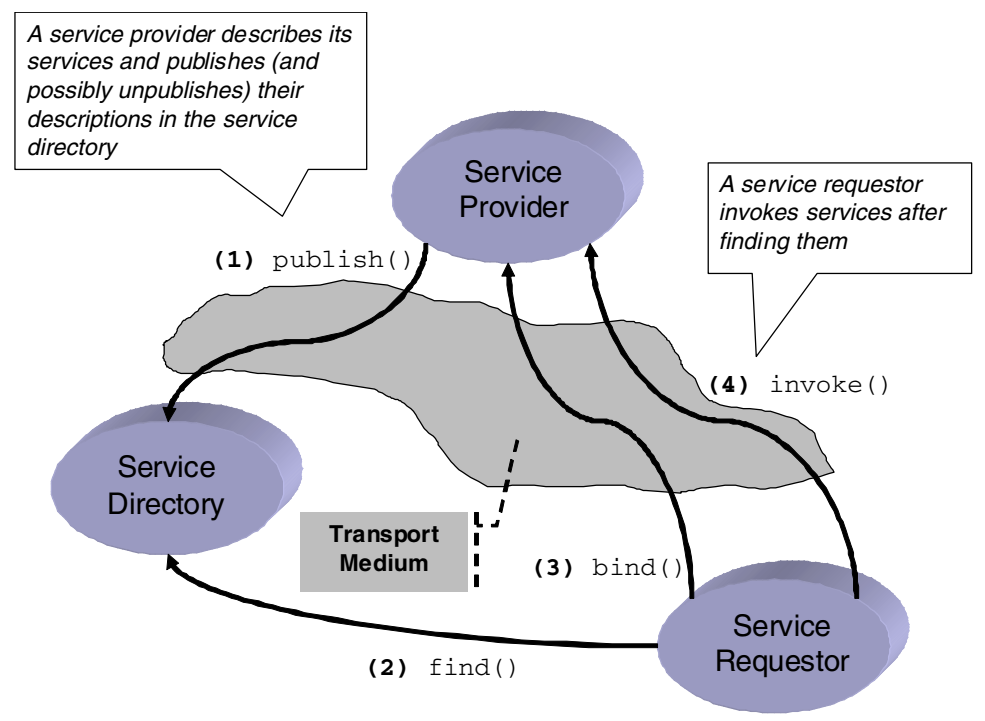

Fig. 2. Basic elements of an e-Service framework

- Service Provider: it is the subject providing software applications for specific needs as services; (i) from a business perspective, this is the owner of the service (e.g., the subject which is possibly paid for its services), and 
(ii) from the architectural perspective, this is the platform the service is deployed onto. Available services are described by using a service description language and advertised through the publish() operation on a public available service directory.

- Service Requestor: it is the party that uses the services; (i) from a business perspective, this is the business requiring certain services to be fulfilled (e.g., the payer subject), and (ii) from an architectural perspective, this is the application invoking the service. A service requestor discover the most suitable service in the directory through the find() operation, then it connects to the specific service provider through the bind () operation and finally it uses it (invoke () operation).

- Service Directory: it is the party providing a repository and/or a registry of service descriptions, where providers publish their services and requestors find services.

As the transport medium is a parameter of a SOA, this framework is easily integrable on different technologies and then well suited for open and dynamically changing environment as the Web is.

Currently, some competing solutions for $e$-Services and Web Services are emerging in commercial contexts, such as the Universal Description, Discovery and Integration (UDDI) $\sqrt{2}$ initiative, and the ebXML 3 standardization effort. All such proposals, on the basis of a common transport medium, consisting of Web technologies such as HTTP, SOAP and XML Protocol, address some basic technological issues of a SOA, that is the definition (i) of the service directory, (ii) of the service description language, (iii) of how to define possible interactions a service can be involved in (conversations), and (iv) of how to compose and coordinate different services, to be assembled together in order to support complex processes (orchestration).

In the UDDI initiative, the architecture of a distributed service directory is proposed 10]; many service description languages are being proposed for specific purposes:

- Web Service Description Language (WSDL, 11]) for describing services, specifically their static interfaces; on top of it, a specific ontology-based language for $e$-Services has been proposed, namely DAML-S [12];

- Web Service Conversation Language (WSCL, [13]) for describing the conversations a service supports;

- Web Service Choreography Interface (WSCI, 14]) for describing the observable behavior of a service in terms of temporal and logical dependencies among the exchanged messages;

- Web Service Flow Language (WSFL, [15]) for the design of composite Web Services starting from simple ones (composition of Web Services);

- XLANG 16 for both the specification of the behavior of services and their orchestration;

- Business Process Execution Language for Web Services (BPEL4WS, [17) with the aim of merging WSFL and XLANG.

${ }^{2}$ UDDI.org: http: //www .uddi .org.

3 ebXML.org: http://www . ebxml .org. 
The architecture of EU-PUBLI.com, outlined in the next section, is based on the previous technologies, that are adopted and homogeneously integrated in an $e$-Government framework.

\section{The EU-PUBLI.com Architecture}

The EU-PUBLI.com project attempts to achieve cooperation amongst European agencies by designing and implementing a cooperative system that can interconnect, at application level, the different information systems, in order to (semi-) automate inter-country macro-processes providing complex $e$-Government services; as an example, in the project demonstrator, the macro-process of establishing in Greece an affiliated company (i.e., an independent "branch office") of an Italian company will be experimented.

Figure 3 shows the overall EU-PUBLI.com architecture; the basic vision consists on defining an overall architecture, respecting the autonomy of the single involved agency; ad hoc development and integration are feasible, but they will produce complex distributed application difficult to maintain and evolve. Instead, the choice of a SOA ensures an high level of flexibility to the system.

The cooperation of different agencies is achieved by making them responsible for exporting some views of its own information system as $e$-Services; the Cooperative Gateway sub-system represents "where" and "how" $e$-Services are deployed; it includes the definition on how different cooperating organizations are organized and connected and how pre-existing legacy applications (Local IS in the figure) can be integrated in a common cooperative process. Roughly speaking, it exports the set of data and application services offered by a single agency through well-defined interfaces. In the architecture, each cooperating agency offers its own cooperative gateway. Clearly, cooperative gateways has the role of service provider in the SOA.

$e$-Services exported on cooperative gateways can be either informative $e$ Services, i.e., providing only static information (stage 1 and 2 of the ANAO model) or transactional $e$-Service, i.e., allowing dynamic retrieve of data and update on back-end databases (stage 3 and 4).

The Orchestration Engine sub-system is the responsible of coordinating all the $e$-Services involved in a cooperative process: through "cooperative process definitions" (technically referred to as orchestration schemas) stored into the Information Manager, it dynamically finds and links suitable $e$-Services. In some sense, this sub-system can be viewed as a particular service requestor of the SOA.

The core of the architecture is represented by the Information Manager: this sub-system stores both (i) e-Service definitions and (ii) orchestration schemas. Moreover, it stores and manages all information needed to convert and map different legal frameworks.

In general, it is accessed at design-time by the cooperating agencies, in order to publish and register their $e$-Services, and to define and register the cooperative schemas with the needed mappings. The information manager is also accessed at run-time by the orchestration engine, in order to gain access to both $e$-Services specifications and instance data, and to update running process instance data. In next section it will be discussed how this sub-system supports knowledge about 


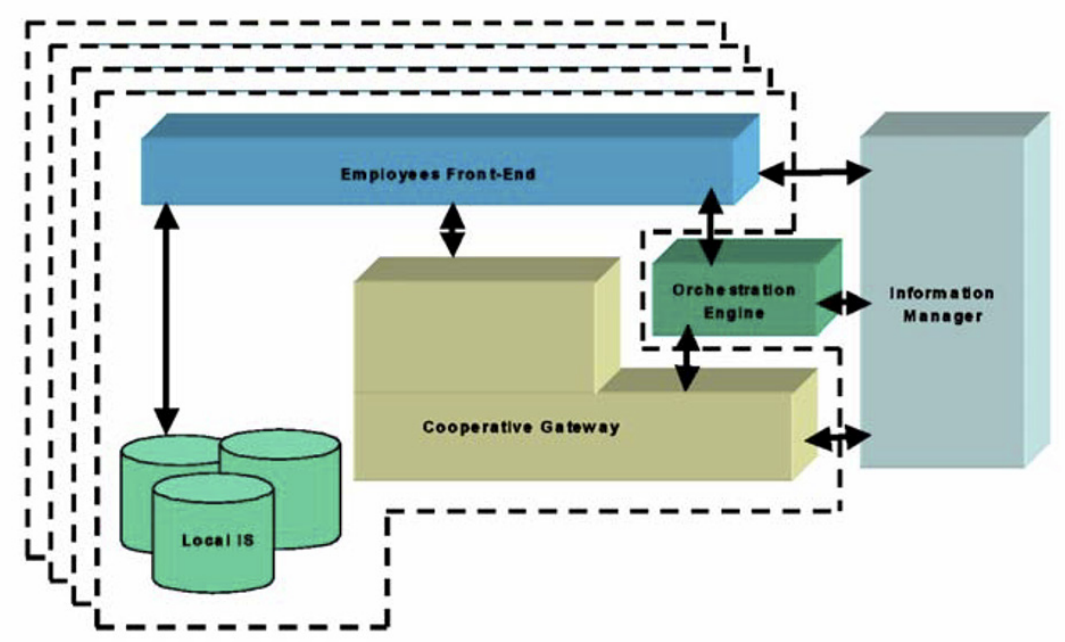

Fig. 3. EU-PUBLI.com Architecture

cooperative processes. With respect to the SOA, the EU-PUBLI.com information manager plays the service directory role.

Finally the Employees Front-End sub-system is responsible for the presentation to end users of the results of cooperative process executions.

\section{Knowledge Management in EU-PUBLI.com}

As discussed in the previous sections, building cooperative process applications requires the integration of different heterogeneous systems, which are different not only from the technological point of view but also for the information asset they export. Whatever the progress in ICT could afford a syntactic interoperability among the PA information systems, an effective and durable cooperation, founded on the dynamic sharing of information and services, can be automated via electronic channel only after the achievement of a semantic agreement, among the interacting parties, about the meaning of the information and services exchanged. Thus, a relevant aspects to be dealt with, for a real cooperation among different agencies, is the knowledge management, at least at level of definition and use of explicit knowledge.

In the last years the problem of the semantic agreement on the information and services interleaved in a cooperative process is being approached through the definition of atomic semantic entities, the ontologies, whose meaning is globally defined and shared within a community. Through the ontologies, each participant of the community can make use of this basic building block to describe its own information and knowledge asset. The definition and the adoption of such a semantic reference model can thus automatize the knowledge mapping within the community; moreover it can represent the starting point for further communitywide automatic composition and retrieval of other knowledge. 
What the EU-PUBLI.com architecture represents is a technological solution which is able to realize an overall cooperative information system spanning national and cross-country agencies. Its adoption requires a deep analysis phase, aimed to identify macro-processes, followed by a formalization phase of all the collected information. These steps produce a coherent and homogeneous documentation of the business practices that are often scattered and rarely formally documented. A proper formalization and representation of the collected information through XML dialects and their storage in the Information Manager sub-system are actually mandatory operation to the correct run of the Orchestration Engine.

In such a framework, through the definition of additional appropriate informative $e$-Services interfacing the Information Manager, the overall architecture can provide the functionalities of an electronic library from which old, current or specific administrative procedures can be retrieval.

Moreover, the Orchestration Engine produces data on effective run-time executions, bottlenecks, etc., that can be stored, analyzed and mined in order to infer process knowledge [4] and to enhance a deeper comprehension of each public agency role within both the national and the cross-country public service scenario. A deeper knowledge of the causal relation among the several activities will become, in an iterative fashion, the starting and the target point for process reengineering involving not only the information system reengineering but even legal constraints; in this cycle the $e$-Governance and the the Electronic Service Delivery become overlapping disciplines.

At current stage of our project and investigation it is not yet clear whether the description of the signature and the behaviour of the involved $e$-Services is a sufficient description, or also dictionaries of each single involved data are required. Currently both $e$-Service descriptions and orchestration schemas in the EU-PUBLI.com architecture are based on specific XML dialects, and this is coherent with the DAML approach to ontology definitions; therefore extensions to more sophisticated techniques should be simple.

\section{Related Work}

The problem of inter-agency cooperation at European level has been already emerged in several different research contexts, as, for example, in the IST FASME project [18. The project focused on the integration of an European citizen with many European PA's through the prototyping of a set of services relying on the technological support of Internet and smartcards.

In the project, one of the considered services was the residence registration service by mobile Europeans at local administrations. What emerged was that the registration procedure generically require information about one' own birth, marriage and children, but these concepts does not have neither a Europeanwide representation nor a European-wide meaning: (i) the certificates attesting these kind of information, have in each country its own different format and data contents; (ii) taking as example the marriage, it could be referred to monogamy, polygamy, polyandry or same-sex marriage according to the country in which the concept is instantiate. 
A crucial point for the effective realization of ICT-aided cooperation among European PA's is, thus, the cross-countries information exchange. Actually, as a legal concept, generally spans a legal framework ruling several different rights (as in the case of marriage, the tax payment and inheritance, for instance) the project strengthen that to prevent legal confusion it was - and it still is - important to agree on the meanings of the information explicitly or implicitly exchanged, also because a specific aspect caught in a country could not exist or could be even explicitly not recognized in other countries.

To face these problems the FASME project relied on standardized "template solution" derived from a legal and administrative harmonization at European level. By following such an approach the transition to $e$-Government solutions might be hampered by the need of a deep business process reengineering moving towards an hardly, slowly and unlikely reachable common legal framework. A more feasible approach to integration and cooperation at European level should, instead, (i) preserve different culture, ideologies and legal framework and (ii) detect, collect or even trigger commonalities and harmonizations at process level among two or more national legal framework.

In this view, the EU-PUBLI.com architecture could represent the information repository where to store cross-nation agreements and partnership; as an example, in the demonstrator we are investigating, the Information Manager will store and manage the mapping rules among those certificates and forms in the two different pilot countries, which are needed for the business establishment. Compared with a static template solution, a framework in which process and data schemas are dynamically loaded and linked together to reflect well-defined administrative provisions, could actually facilitate and accelerate the actuation of cooperative $e$-Government initiatives. Such a widely configurable solution can be flexibly queried and enriched to reflect social evolution and trigger legal changes.

Along the way towards the definition of common metadata and data schemas definition, an important experience is the one related with the British eGovernment Interoperability Framework (e-GIF) project [19, in which the issue has being faced with a centralized approach relying on XML technologies. The $e$-GIF currently comprises a wide set of XML documents, publicly available, defining mandatory technical policies and specification (i) for achieving interoperability and data integration; (ii) for ruling information access and content management and thus (iii) for ensuring information system coherence across the British public sector.

The XML schemas definition process is realized through an interactive and iterative procedure: (i) proposals and revisions for an XML schema involve a central coordination team but also all the stakeholders so to ensure its wide acceptance; (ii) the order in which the XML schemas are defined or refined is driven by the need of citizens.

The $e$-GIF initiative is claiming more and more interests and it could represent an important methodological and technical reference for the next step along the way to a real adoption and and effective use of approaches and architectures similar to the one investigated in the EU-PUBLI.com project. 


\section{Conclusions}

In this paper, we have presented the EU-PUBLI.com architecture, specifically focusing on $e$-Government service provisions based on orchestration of $e$-Services. The basic concept of our approach is the one of cooperative process, as unifying element among different inter-country agencies providing value-added services to European citizens.

Moreover, we have discussed how the proposed architecture support a form, although simple, of knowledge management, specifically focused on cooperative processes:

- all the information collected in the analysis phase, aimed to identify the cooperative process, produces a coherent and homogeneous documentation of the business practices. Such a documentation (i) must be formalized in an appropriate manner to be stored as part of the supporting architecture and to drive the orchestration of the different $e$-Services (e.g., in the case of the mappings among different legal frameworks); and (ii) can be later offered to citizens and employees through informative $e$-Services;

- orchestration of $e$-Services (i.e., the enactment of the cooperative processes) produces information on effective run-time executions, exceptions, bottlenecks, etc.; such information can be analyzed and mined in order to infer new process knowledge.

Focusing on processes, on the other hand, tends to underestimate the importance of automatizing the knowledge mapping and the automatic composition and retrieval of other knowledge, which conversely are addressed by ontologybased techniques. Therefore an important issues that need to be resolved in order to develop a complete $e$-Government framework, is how an ontology-based approach to knowledge management can be integrated and complemented with the one based on cooperative process execution and $e$-Service orchestration proposed in this paper.

Acknowledgments. This work has been supported by the European Commission under Contract No. IST-2001-35217, Project EU-PUBLI.com (Facilitating Cooperation amongst European Public Administration Employees) (http://www.eu-publi.com/). The authors would like to thanks the other project partners: CERTH/ITI (Greece), ICE (Italy), ALTEC (Greece) and IBERMATICA (Spain) for discussing many issues dealt in this paper.

\section{References}

1. C. Batini and M. Mecella, "Enabling Italian $e$-Government Through a Cooperative Architecture", IEEE Computer, vol. 34, no. 2, 2001.

2. M. Mecella and B. Pernici, "Designing Wrapper Components for $e$-Services in Integrating Heterogeneous Systems", VLDB Journal, vol. 10, no. 1, 2001.

3. M. Mecella, F. Parisi Presicce, and B. Pernici, "Modeling $e$-Service Orchestration Through Petri Nets", in Proceedings of the 3rd VLDB International Workshop on Technologies for e-Services (VLDB-TES 2002), Hong Kong, Hong Kong SAR, China, 2002. 
4. D. Grigori, F. Casati, U. Dayal, and M.C. Shan, "Improving Process Quality through Exception Understanding, Prediction and Prevention", in Proceedings of the 27th International Conference on Very Large Data Bases (VLDB 2001), Roma, Italy, 2001.

5. R.E. Freeman, Strategic Management: a Stakeholder Approach, Pitman, 1984.

6. M.E. Porter and V.E. Millar, "How Information Gives You Competitive Advantage", Harvard Business Review, 1985.

7. Australian National Audit Office, "Electronic Service Delivery, including Internet Use, by Commonwealth Government Agencies", The Auditor-General, Audit Report No.18 (1999-2000), 1999.

8. Central IT Unit of the Cabinet Office (CITU), "Information Age Government. Benchmarking Electronic Service Delivery", CITU Report, London, United Kingdom, July 2000.

9. T. Pilioura and A. Tsalgatidou, "e-Services: Current Technologies and Open Issues", in Proceedings of the 2nd VLDB International Workshop on Technologies for e-Services (VLDB-TES 2001), Rome, Italy, 2001.

10. UDDI.org, "UDDI Technical White Paper", http://www .uddi.org/pubs/lru_UDDI_Technical_Paper.pdf, 2001.

11. Ariba, Microsoft, and IBM, "Web Services Description Language (WSDL) 1.1", W3C Note. http://www.w3.org/TR/2001/NOTE-wsdl-20010315, March 2001.

12. A. Ankolekar, M. Burstein, J. Hobbs, O. Lassila, D. Martin, D. McDermott, S. McIlraith, S. Narayanan, M. Paolucci, T. Payne, and K. Sycara, "DAML-S: Web Service Description for the Semantic Web", in Proceedings of the 1st International Semantic Web Conference (ISWC 2002), Chia, Sardegna, Italy, 2002.

13. H. Kuno, M. Lemon, A. Karp, and D. Beringer, "Conversations + Interfaces = Business Logic", in Proceedings of the 2nd VLDB International Workshop on Technologies for e-Services (VLDB-TES 2001), Rome, Italy, 2001.

14. BEA, Intalio, SAP, and Sun, "Web Service Choreography Interface (WSCI) 1.0", Document.

http: //wwws. sun.com/software/xml/developers/wsci/wsci-spec-10.pdf, 2002.

15. F. Leymann, "Web Service Flow Language (WSFL 1.0)", IBM Document. http://www-4.ibm.com/software/solutions/webservices/pdf/WSFL.pdf, May 2001.

16. T. Satish, "XLANG. Web Services for Business Process Design", Microsoft Document. http://www.gotdotnet.com/team/xml_wsspecs/xlang-c/default.hm, 2001.

17. F. Curbera, Y. Goland, J. Klein, F. Leymann, D. Roller, S. Thatte, and S. Weerawarana, "Business Process Execution Language for Web Services (Version 1.0)", IBM Document. http://www.ibm.com/developerworks/library/ws-bpel/, July 2002.

18. FASME Project: http://www.fasme.org.

19. Office of the $e$-Envoy, " $e$-Government Interoperability Framework", 2002. 